\title{
FUERZA DE MORDEDURA Y ESTRÉS MANDIBULAR EN EL JAGUAR (PANTHERA ONCA) DURANTE LA DEPREDACIÓN DE PECARÍES (ARTIODACTYLA: TAYASSUIDAE) MEDIANTE LA FRACTURA DE SUS CRÁNEOS
}

\author{
J. Fernando Del Moral Sachetti, ${ }^{1 *}$ Fátima I. Lameda Camacaro, ${ }^{1}$ Jorge \\ Santiago VÁzQuez ${ }^{2}$ \& Ramiro Zenteno Cárdenas ${ }^{3}$ \\ ${ }^{1}$ Fundación AndígenA, Mérida, Venezuela.

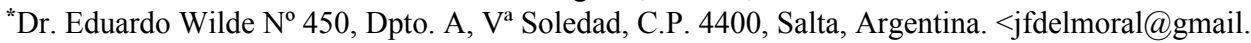 \\ com> \\ ${ }^{2}$ Zoológico Miguel Álvarez del Toro, Calzada Cerro Hueco sin número, Colonia El Zapotal, C.P. \\ 29000, Tuxtla Gutiérrez Chiapas, México. \\ ${ }^{3}$ Fundación Biodiversa Bolivia, Santa Cruz de la Sierra, Bolivia.
}

Del Moral Sachetti, J. F., F. I. Lameda Camacaro, J. S. Vázquez \& R. Zenteno Cárdenas. 2011. Fuerza de mordedura y estrés mandibular en el jaguar (Panthera onca) durante la depredación de pecaríes (Artiodactyla: Tayassuidae) mediante la fractura de sus cráneos. Acta Zoológica Mexicana (n. s.), 27(3): 757-776.

RESUMEN. Una de las formas de depredación más efectivas del jaguar (Panthera onca) consiste en la aplicación de su fuerza de mordedura en el neurocráneo de los pecaríes, una de sus principales presas naturales, provocándole un estrés altamente compresivo que deviene en la posterior fractura del cráneo con una muerte rápida. En el presente estudio se analizaron mediante el método del cráneo seco, 15 cráneos completos y medidas craneométricas reportadas en la literatura, para jaguares adultos pertenecientes a diferentes subespecies o fenotipos del rango distributivo de $P$. onca. Afín de obtener sus fuerzas de mordedura canina y carnasial, esfuerzos de corte y momentos flexores máximos a lo largo del vano mandibular. La obtención de estas variables, en una estructura hiperestática se efectúo mediante el Método de Aproximaciones Sucesivas de Cross. Después se correlacionaron las fuerzas de mordedura máximas del jaguar y su acción en la sección transversal del neurocráneo de Pecari tajacu, Tayassu pecari y Catagonus wagneri mediante el método de Cremona, para obtener las tensiones internas que someten al cráneo de los pecaríes previa fractura. Se encontró que la fuerza máxima de mordedura canina del jaguar es de 681,56 Newton siendo 3 veces más compresiva en los carnasiales. Estas son lo suficientemente altas, como para provocar la rotura de una estructura rígida, como la sección triangular del neurocráneo en los pecaríes. Además los caninos robustos del jaguar resisten las fuerzas de pandeo aplicadas sobre los mismos durante la lucha con la presa, y una boca más amplia ayuda a una mejor adherencia y distribución uniforme de las fuerzas de mordedura, durante la mordida mortal.

Palabras Claves: Biomecánica, jaguar, pecaríes, dentición, morfología.

Recibido: 03/03/2011; aceptado: 10/08/2011. 
Del Moral Sachetti, J. F., F. I. Lameda Camacaro, J. S. Vázquez \& R. Zenteno Cárdenas. 2011. Bite force and jaw stress in the jaguar (Panthera onca) during predation of the peccaries (Artiodactyla: Tayassuidae) by fracture of its skulls. Acta Zoológica Mexicana (n. s.), 27(3): 757-776.

ABSTRACT. One of the most effective ways of the jaguar's predation (Panthera onca) is the application of bite force at the neurocranium of peccaries, one of its main natural preys, causing highly compressive stress that becomes fracture with its back a quick killing. In the present study were analyzed by the method of dry skull, 15 adult jaguar's skulls moreover completing craniometrical measures with reported data in the literature belonging to different subspecies or phenotypes of this species' distributional range, to obtain its maximum bite's force canines and carnassials moreover of the cutting efforts and maximum flexor moments along the jaw. The resolution of these variables in a hiperestaticity structure is achieved through the Moment Distribution Method by Cross. After correlated the maximum bite forces of the jaguar and its action in the cross section of the neurocranium of Pecari tajacu, Tayassu pecari and Catagonus wagneri by Cremona method to obtain the internal stress for before skull fracture. It was found that jaguar's maximum canine bite force is of 681.56 Newton and in the carnassials line is still 3 times most compressive. These forces are sufficiently high to cause fracture of a rigid structure as is the neurocranium's triangular section of the peccaries. Moreover the jaguar's robust canines resist the bending forces applied by struggling prey and a wider muzzle helps to stabilize grip and distribute bite forces more evenly during the killing bite.

Key words: Biomechanics, jaguar, peccaries, dentition, morphology.

\section{INTRODUCCIÓN}

El jaguar (Panthera onca), es el mayor félido de la región neotropical y el único representante de la subfamilia Pantherinae en el continente americano. Considerado por varios autores como un carnívoro oportunista (véase Rabinowitz \& Nottingham 1986, Emmons 1987, Seymour 1989, Sunquist \& Sunquist 2002) básicamente predador de mamíferos, y de esta clase los ungulados son las principales presas de $P$. onca en términos de biomasa (Schaller 1983, Emmons 1992, Aranda 1994, González-Maya 2007, Hoogesteijn \& Hoogesteijn 2008). En este sentido, varios autores que han estudiado la alimentación del jaguar en vida silvestre, mencionan entre los ungulados, particularmente a los pecaríes como presas significativas (Rabinowitz \& Nottingham 1986, Emmons 1987, Aranda 1992, 1994, Chinchilla 1994, Sunquist \& Sunquist 2002, González-Maya 2007, Salom-Pérez et al. 2007). A pesar que esta preferencia no está influenciada necesariamente con la disponibilidad de los pecaríes en el hábitat del jaguar (Aranda 1994, Chinchilla 1994, Carrillo 2000, Crawshaw \& Quigley 2002, González-Maya 2007, Salom-Pérez et al. 2007).

Actualmente se reconocen tres especies de pecaríes en el Neotrópico: el pecarí de collar Pecari tajacu, el pecarí labiado Tayassu pecari y el pecarí del Chaco Catagonus wagneri (Grubb \& Groves 1993, Grubb 2005). Las dos primeras especies mencionadas sobreponen casi totalmente su distribución con la de $P$. onca, mientras que C. wagneri endémico de la región fitogeográfica del Chaco semiárido (bosque caducifolio-xérico que se distribuye parcialmente por Paraguay, Bolivia y Argentina) además de simpátrico con las otras dos especies de pecaríes en esta región (Wetzel 1977), sobrepone también su distribución reducida con la del jaguar. Sin embargo de las tres 
especies, solo el pecarí de collar y el pecarí labiado constituyen presas importantes para $P$. onca (véase Aranda 1992, 1994, Chinchilla 1994, Carrillo 2000, Crawshaw \& Quigley 2002, Sunquist \& Sunquist 2002, González-Maya 2007, Salom-Pérez et al. 2007, Cavalcanti 2008).

Aranda (1994) discute asimismo, que la complexión robusta del jaguar (Fig. 1a) en comparación a otros pantherines, podría explicarse en una adaptación a la depredación preferente hacia los pecaríes. Como en menor medida para producir la rotura de integumentos duros de armadillos, tortugas y caimanes (Schaller \& Vasconcelos 1978, Emmons 1989, Sunquist \& Sunquist 2002, Meachen-Samuels \& Van Valkenburgh 2009). Considerando que los pecaríes son de tamaño mediano (con una masa corporal $\leq 45 \mathrm{~kg}$ ) y tienen prominentes caninos resistentes a gran presión mandibular (véase Kiltie 1982), además de hábitos gregarios y de autodefensa que le permiten contraatacar en grupo para contrarrestar cualquier evento predatorio del carnívoro. Pudiendo ocasionarle inclusive serias heridas al predador, esto refleja que los pecaríes no son una presa fácil para el jaguar (Aranda 1994, Crawshaw \& Quigley 2002, Salom-Pérez et al. 2007). Sin embargo no se tienen mayores evidencias para el caso, aunque es conocida la gran fuerza de mordedura del jaguar en relación a su masa corporal (véase Wroe et al. 2005, Christiansen 2008, Meachen-Samuels \& Van Valkenburgh 2009), como la amplitud de sus maxilares y dentarios con un rostro relativamente corto; y la línea de acción de sus músculos aductores que le confieren también una gran ventaja mecánica, permitiéndole asirse con facilidad de la presa al morder (Ewer 1973). Además de los caninos robustos de $P$. onca que son resistentes a la flexión axial (Fig. 1b, véase Freeman \& Lemen 2007, Meachen-Samuels \& Van Valkenburgh 2009), producto de la compresiva mordedura mortal que provoca frecuentemente la rotura de la base craneana de la presa o de las vértebras cervicales y de la médula espinal (Brock 1963, Leyhausen 1979, Schaller \& Crawshaw 1980, Rabinowitz 1986, Crawshaw \& Quigley 2002). Confiriéndole estas características en conjunto, alta eficiencia durante la depredación. En este sentido mediante el presente estudio, nosotros hipotetizamos que la fuerza de mordedura del jaguar responde al particular comportamiento predatorio que tiene $P$. onca, hacia sus presas principales. Unidireccionalmente, se analiza la morfología y mecánica craneodentaria del jaguar correlacionada con la depredación de las tres especies de pecaríes (P. tajacu, T. pecari, $C$. wagneri) mediante la mordedura y fractura de sus cráneos. Para estimar en $P$. onca, la fuerza de mordedura y el estrés mandibular; así también, para la obtención de las tensiones internas en los cráneos de pecaríes, se ha empleado el "método del cráneo seco" (Thomason 1991, Wroe et al. 2005, Christiansen \& Wroe 2007, Del Moral et al. 2009). Para estudiar la mecánica de mordedura, se utilizó el modelo de la mandíbula como una viga en voladizo, con el fin de estimar la carga máxima actuante en diferentes locus del dentario (Dechow \& Carlson 1983, Badoux 1986). Este enfoque ha sido empleado en varias especies de mamíferos y permite obtener un perfil de 


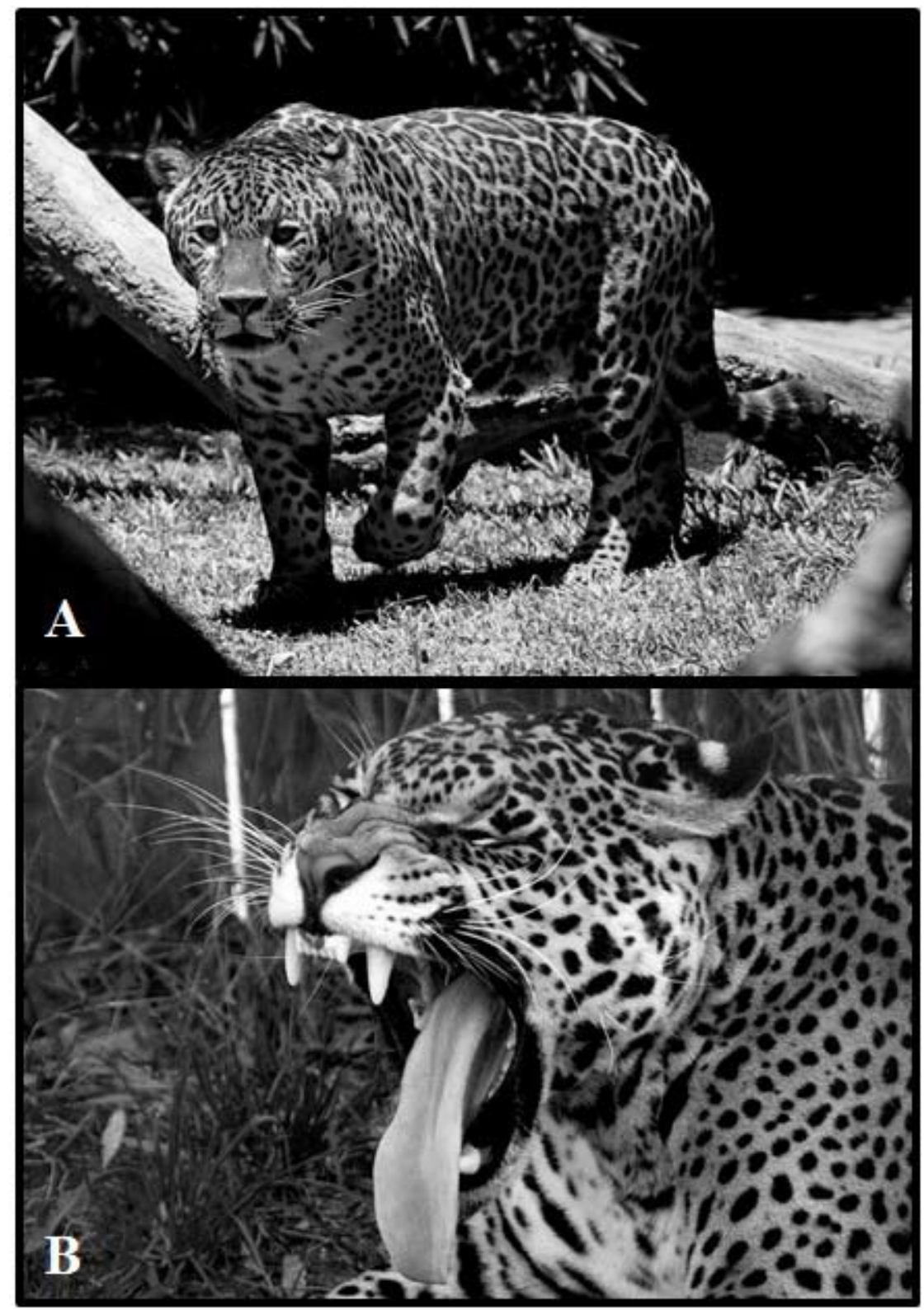

Figura 1. A) El jaguar (Panthera onca), es el único representante de la subfamilia Pantherinae en América. $P$. onca tiene una complexión física robusta en relación a su masa corporal, comparado con pantherines del Viejo Mundo. B) Los caninos son una característica de todos los carnívoros, que además de ser utilizados en el comportamiento agonístico, son las armas más importantes para someter presas en la mayoría de los grupos. Crédito fotográfico: Belén Etchegaray. 
la fuerza mandibular (véase Dechow \& Carlson 1983, Binder \& Van Valkenburgh 2000, Therrien 2005, Christiansen 2007, 2008). No obstante a diferencia de estos estudios citados, cuyo punto de vista fue isostático, nosotros abordamos el análisis desde una perspectiva hiperestática que se asemeja más a la realidad de la problemática (Del Moral et al. 2009).

\section{MATERIALES Y MÉTODOS}

Datos de la muestra y protocolo de análisis. Se estudió una muestra total de 15 cráneos completos de jaguares adultos provenientes de vida silvestre $(\geq 2,5$ años de edad, i.e. determinado a ojo libre mediante el análisis del desgaste dentario y el desarrollo de la cresta sagital) que incluía aleatoriamente machos $(\mathrm{n}=7)$ y hembras $(\mathrm{n}$ $=8$ ), procedentes de Argentina (pertenecientes a la subespecie P.o. palustris sensu Pocock 1939, $\mathrm{n}=7$ ), Bolivia (P.o. palustris, $\mathrm{n}=4)$, México (P.o. goldmani, $\mathrm{n}=3$ ) y Perú (P.o. peruviana, $\mathrm{n}=1$ ) depositados en diversas colecciones tanto personales, museológicas y zoológicas. En Argentina se estudiaron cinco especímenes que pertenecen a la Estación Biológica de Fauna Silvestre Dr. Martín Vucetich (EBFSMV) de la Universidad Nacional de Jujuy y los restantes dos pertenecientes a la colección personal del primer autor (J. F. Del Moral) ninguno cuenta con número de inventario pero si con datos de colecta, respectivamente. En Bolivia se examinaron los cráneos (CBF2979, CBF1486 y CBF4078) de la Colección Boliviana de Fauna (CBF) de La Paz; y un cráneo completo en exhibición con datos de colecta, pero no inventariado del Museo de Historia Natural Noel Kempff Mercado (MHNNKM) en Santa Cruz de la Sierra. Los ejemplares estudiados en México pertenecen al Zoológico Miguel Álvarez del Toro (ZooMAT, Tuxtla Gutiérrez Chiapas), estos contaron con datos de colecta, pero tampoco se encuentran inventariados. Respecto a Perú se analizó un espécimen sin datos ni código, perteneciente al Departamento de Paleontología de Vertebrados del Museo de Historia Natural de la Universidad Nacional Mayor de San Marcos (MHNUNMSM) de Lima. Los cráneos de pecaríes estudiados pertenecieron a ejemplares adultos y sub-adultos de $P$. tajacu $(\mathrm{n}=5)$, $T$. pecari $(\mathrm{n}=6)$ y $C$. wagne$r i(\mathrm{n}=4)$, provenientes en su totalidad de la colección personal del primer autor en Argentina y colectados en este país. Para el análisis biomecánico de los cráneos de jaguares y pecaríes se empleó el "método del cráneo seco" (Thomason 1991) derivado de la relación entre las dimensiones propias del cráneo y las áreas transversales de los músculos masticatorios, considerando a la mandíbula funcionalmente como un voladizo. En consecuencia ha sido estimado el modelo del voladizo, como un predictor significativo del comportamiento predatorio de mamíferos carnívoros (véase Wroe et al. 2005, Del Moral et al. 2009). En este sentido para los jaguares, se seleccionaron 10 medidas cráneo dentarias (Fig. 2a), tomadas en base al diseño genérico de la caja craneana (afectada a las líneas de acción de los músculos masticatorios) y el dentario de la especie (Del Moral et al. 2009). Para la medición se empleó un Vernier de 


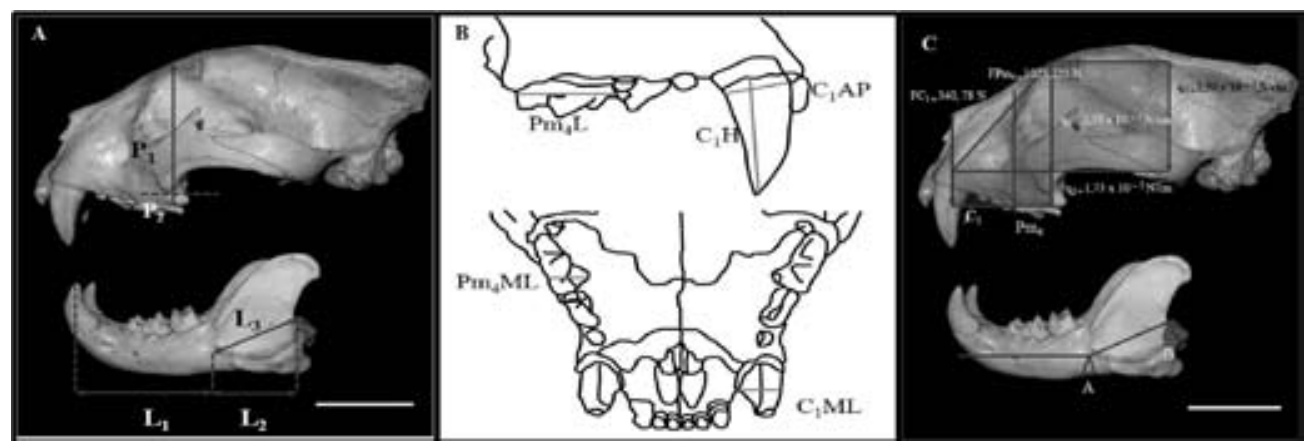

Figura 2. Diagrama de medidas craneales, mandibulares, dentarias y sistemas de cargas del cráneo de Panthera onca, empleadas en este trabajo (fotografías tomadas de Aparicio \& López Plana 2011). A) Vista lateral del cráneo y dentario. B) Dibujo de la vista lateral del maxilar y la dentición; y vista palatina con las respectivas mediciones dentarias. C) Cargas puntuales $\left(\mathrm{FC}_{1} ; \mathrm{FPm}_{4}\right)$, uniformemente distribuidas triangular $\left(\mathrm{q}_{1}\right)$ y rectangulares $\left(\mathrm{q}_{2}, \mathrm{q}_{3}\right)$. Para las abreviaciones, véase el texto. Escala de barra $10 \mathrm{~cm}$.

$0,05 \mathrm{~mm}$ de precisión. En la categoría del dentario se consideraron las medidas, $\mathrm{L}_{1}$ : longitud canino-fosa masetérica; $\mathrm{L}_{2}$ : longitud inicio fosa masetérica-apófisis angular y $\mathrm{L}_{3}$ : luz inicio fosa masetérica-apófisis condilar. En la categoría alturas en la caja craneana se consideraron las medidas, $\mathrm{P}_{1}$ : altura de la caja craneana desde el frontal (en adyacencias del proceso supraorbital) -porción distal de la línea masetérica del yugal y $\mathrm{P}_{2}$ : altura desde el extremo distal de la línea masetérica del yugal hasta el extremo distal del maxilar. También se tomaron las siguientes medidas dentarias respecto del canino $\left(\mathrm{C}_{1}\right)$ y el 4 to premolar $\left(\mathrm{Pm}_{4}\right)$ superiores (Fig. $\left.2 \mathrm{~b}\right), \mathrm{C}_{1} \mathrm{H}$ : altura del esmalte de $\mathrm{C}_{1} ; \mathrm{C}_{1} \mathrm{AP}$ : diámetro antero posterior de $\mathrm{C}_{1}$ en la unión del esmalte; $\mathrm{C}_{1} \mathrm{ML}$ : diámetro medio lateral de $\mathrm{C}_{1}$ en la unión entre el esmalte y la dentina; $\mathrm{Pm}_{4} \mathrm{~L}$ : longitud antero posterior de $\mathrm{Pm}_{4}$ y $\mathrm{Pm}_{4} \mathrm{ML}$ : ancho medio lateral a través del protocono de $\mathrm{Pm}_{4}$ (Cuadro 1). En tanto para los pecaríes, se consideraron las siguientes dimensiones caudales (Fig. 3a), POE-CO: diagonal desde la protuberancia occipital externa a la porción distal lateral del cóndilo occipital; POE-AY: diagonal desde la protuberancia occipital externa a la porción distal lateral del apófisis yugular; CO-CO: distancia entre extremos de ambos cóndilos occipitales y AY-CO: distancia entre extremos del proceso yugular y el cóndilo occipital próximo a este (Cuadro 2). Para todas las denominaciones óseas o musculares en el tratamiento de ambas especies, se empleó la nomenclatura de la Nómina Anatómica Veterinaria (2005).

Determinación de las fuerzas de mordedura canina y carnasial. En los especímenes de jaguares analizados y además mediante el empleo de variables cráneo dentarias de individuos silvestres (reportadas en la literatura) concernientes a las ocho 


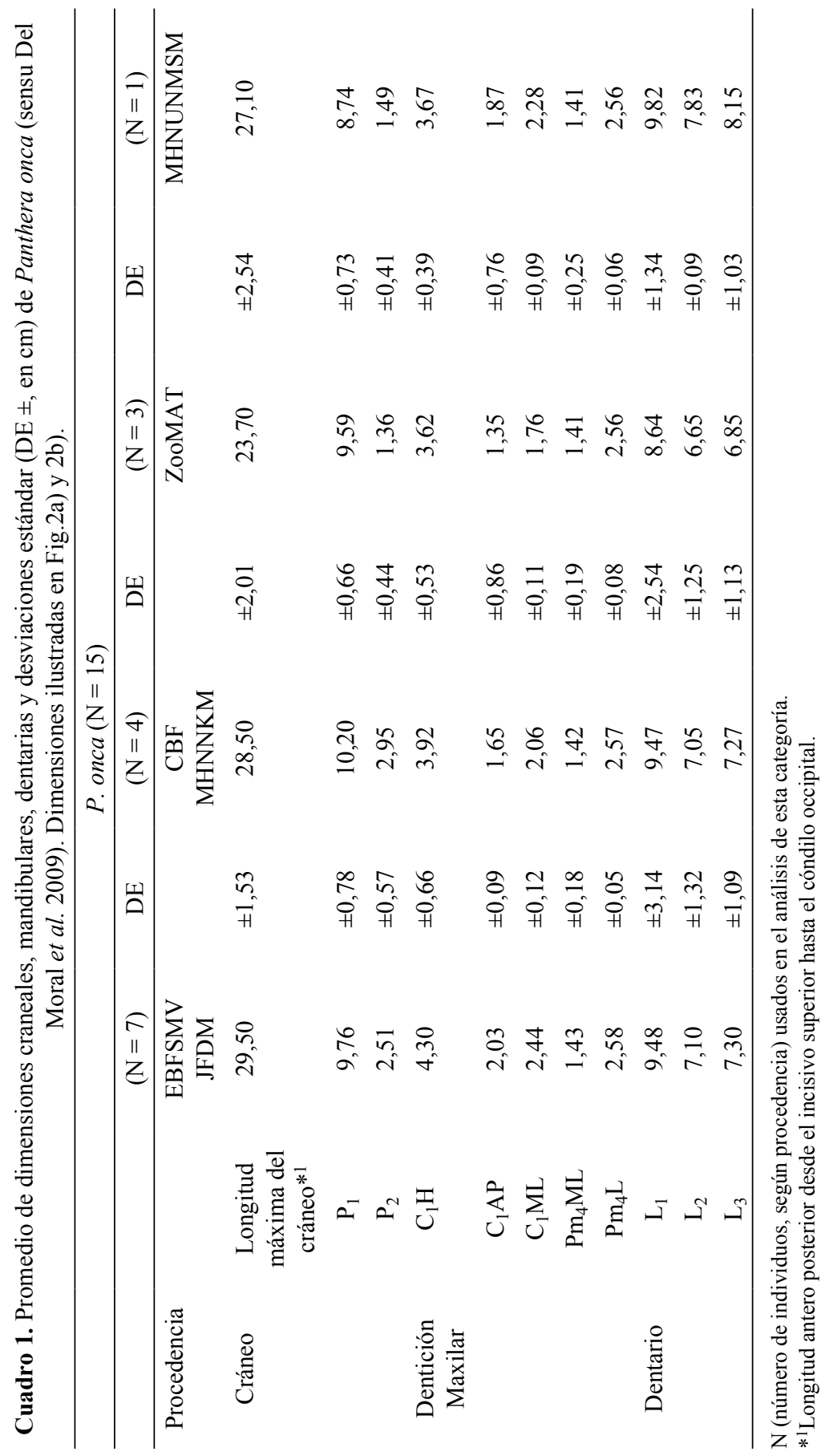



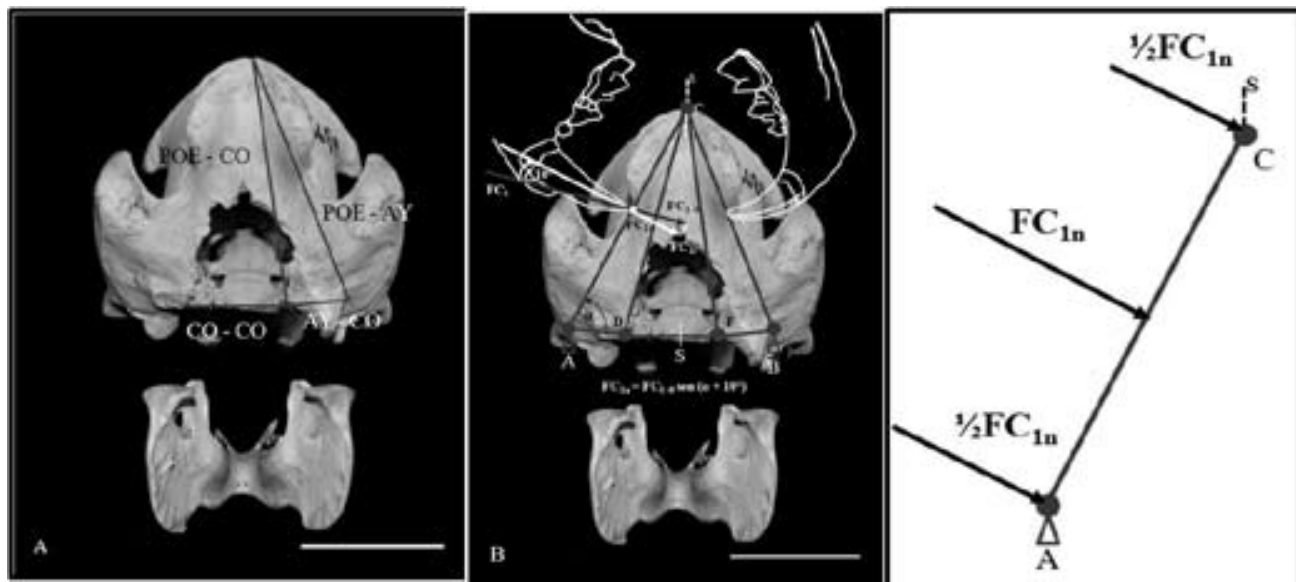

Figura 3. Diagrama de medidas craneales y sistemas de cargas en el neurocráneo de un ejemplar de Tayassu pecari, empleadas en este trabajo (fotografías tomadas de Aparicio \& López Plana 2011). A) Vista caudal del cráneo en los pecaríes y dimensiones consideradas. B) Fuerza canina unilateral $\left(\mathrm{FC}_{1}\right)$ de $P$. onca y descomposición en una presión $\mathrm{FC}_{1 \mathrm{n}}$ perpendicular a la barra POE-AY y otra $\mathrm{FC}_{1 \mathrm{i}}$ tangente a POE-AY $\left(\mathrm{FC}_{1 \mathrm{n}}\right.$ no ejerce presión sobre POE-AY). $\mathrm{FC}_{1 \mathrm{n}}$ se distribuye en partes iguales sobre

los nudos $(\mathrm{A}, \mathrm{C})$ correspondientes. Para las abreviaciones, véase el texto. Escala de barra $10 \mathrm{~cm}$.

subespecies reconocidas por Pocock (1939) y Seymour (1989), definidas en función a patrones diferenciales de pelaje y caracteres morfológicos. Se calcularon las fuerzas de mordedura canina (FMC) y las fuerzas de mordedura carnasial (FMM), en los dos pares caninos $\left(\mathrm{C}_{1}\right)$ y los dos pares carnasiales $\left(\mathrm{Pm}_{4}\right.$ y molar inferior $\left.\mathrm{M}_{1}\right)$ del conjunto cráneo dentario. A tal fin, se emplearon medidas de cráneos pertenecientes a P.o. arizonensis $(\mathrm{n}=2), P$. o. veraecrucis $(\mathrm{n}=2), P$. o. hernandesii $(\mathrm{n}=4), P . o$. goldmani $(\mathrm{n}=4), P$. o. centralis $(\mathrm{n}=6), P$. o. onca $(\mathrm{n}=9), P$. o. peruviana $(\mathrm{n}=6)$ y

Cuadro 2. Promedio de dimensiones caudales y desviaciones estándar ( $\mathrm{DE} \pm$, en $\mathrm{cm})$ del neurocráneo de Pecari tajacu, Tayassu pecari y Catagonus wagneri. Dimensiones ilustradas en Fig.3a).

\begin{tabular}{ccccccc}
\hline Cráneo & P. tajacu $(\mathrm{N}=5)$ & $\mathrm{DE}$ & T. pecari $(\mathrm{N}=6)$ & $\mathrm{DE}$ & C. wagneri $(\mathrm{N}=4)$ & $\mathrm{DE}$ \\
\hline $\begin{array}{c}\text { Longitud } \\
\text { del cráneo*2 }\end{array}$ & 23,50 & $\pm 1,45$ & 25,80 & $\pm 0,64$ & 26,20 & $\pm 0,89$ \\
POE-AY & 7,78 & $\pm 0,25$ & 8,74 & $\pm 0,33$ & 10,70 & $\pm 0,56$ \\
POE-CO & 9,01 & $\pm 0,13$ & 9,55 & $\pm 0,48$ & 11,82 & $\pm 0,67$ \\
AY-CO & 3,05 & $\pm 0,76$ & 2,85 & $\pm 0,65$ & 5,15 & $\pm 0,84$ \\
CO-CO & 3,60 & $\pm 0,87$ & 4,80 & $\pm 0,98$ & 5,24 & $\pm 1,06$ \\
\hline
\end{tabular}

*2Longitud antero posterior desde el incisivo superior hasta el cóndilo occipital.

$\mathrm{N}$ (número de individuos, según especie) usados en el análisis de esta categoría. 
P. o. palustris $(\mathrm{n}=16)$ reportadas por Pocock $(1939)$. También se utilizaron adicionalmente, variables craneanas de especímenes de P.o.goldmani $(\mathrm{n}=20)$ y P.o.onca $(\mathrm{n}=39)$ reportadas por Aranda (1992) y Hoogesteijn \& Mondolfi $(1992,1996)$, correspondientemente. Respecto a las fuerzas de mordedura canina y carnasial, ambas se traducen en las resultantes independientes, producto del estrés compresivo en los complejos caninos y carnasiales durante la mordedura de máxima intensidad. El modelo se consideró en equilibrio, el movimiento y densidad del tejido óseo como una constante, siguiendo premisas básicas de la mecánica estática. FMC se dedujo de la expresión de Eulert sobre la carga crítica de pandeo (véase McMahon 1975, Callister 2000), mientras que FMM queda definida por la ecuación a la compresión simple de la Ley de Hooke (véase Callister 2000), ambas se expresan en Newtons [N]:

$$
\mathrm{FMC}=2\left(\pi^{2} E \mathrm{AC} / c^{2} \mathrm{C}_{1} \mathrm{H}^{2}\right)[\mathrm{N}]
$$

siendo: $E$ (el módulo de elasticidad o módulo de Young) a la compresión del canino $=1054 \mathrm{~N} / \mathrm{cm}^{2}, \mathrm{AC}\left[\mathrm{cm}^{2}\right]$ el área de la sección transversal de $\mathrm{C}, c^{2}=4$ cuadrado del coeficiente de empotramiento y $\mathrm{C}_{1} \mathrm{H}^{2}$ cuadrado de $\mathrm{C}_{1} \mathrm{H}$.

$$
\mathrm{FMM}=2\left(\sigma \mathrm{APm}_{4}\right)[\mathrm{N}]
$$

siendo: la tensión específica $\sigma=1054 \mathrm{~N} / \mathrm{cm}^{2}\left(\right.$ símil a $E$ ) y $\mathrm{APm}_{4}\left[\mathrm{~cm}^{2}\right]$ el área de la sección transversal de $\mathrm{Pm}_{4}$ (a nivel del protocono).

Cálculo de momentos flexores máximos en el jaguar y cálculo del estrés craneal en los pecaríes. El sistema de cargas en el dentario del jaguar, se consideró compuesto por una carga triangular $\left(\mathrm{q}_{1}\right)$ y dos cargas rectangulares $\left(\mathrm{q}_{2}, \mathrm{q}_{3}\right)$ uniformemente distribuidas y dos cargas puntuales $\left(\mathrm{FC}_{1}, \mathrm{FPm}_{4}\right)$. La forma de la carga uniforme, estuvo determinada por el diseño general de la caja craneana y las líneas de acción de la musculatura cráneo mandibular insertas en ésta. La intensidad de las resultantes (Rq) de las cargas uniformemente distribuidas, corresponde al peso propio vivo de la cabeza del jaguar y se estimó en función de la masa corporal y medidas morfométricas de $P$. onca (longitud y altura a la cruz del cuerpo, longitud y altura de la cabeza), obtenidas de la literatura (véase Pocock 1939, Schaller \& Vasconcelos 1978, Seymour 1989, Aranda 1992, Hoogesteijn \& Mondolfi 1992, 1996) y de datos de los autores del presente trabajo. Por lo tanto, $\mathrm{Rq}_{1}$ correspondiente a la carga triangular y $\mathrm{Rq}_{2}$, $\mathrm{Rq}_{3}$ correspondiente a las cargas rectangulares resultan de las siguientes expresiones (véase Del Moral et al. 2009), en función de sus respectivos vanos y luces (L):

$$
\begin{gathered}
\mathrm{Rq}_{1}=1 / 2 \mathrm{q} \mathrm{L}_{1}[\mathrm{~N} / \mathrm{cm}] \\
\mathrm{Rq}_{2}=\mathrm{Rq}_{3}=\mathrm{qL}[\mathrm{N} / \mathrm{cm}]
\end{gathered}
$$


El sistema final propuesto, responde a dos tramos de una estructura hiperestática, en la mandíbula (Fig. 2c): 1) en voladizo desde el canino al apoyo fijo A, situado a nivel del extremo proximal de las fosas masetérica $\left(\mathrm{C}_{1}-\mathrm{A}\right)$, y 2$)$ en barra desde el apoyo articulado B, ubicado en el apófisis condilar al apoyo A (A-B). Por convención de signos se consideran las cargas, negativas $(-)$ y las reacciones a las mismas, positivas $(+)$. El cálculo de los momentos flexores máximos fue expresado en Newtons centímetros $(\mathrm{Ncm})$, en los dos tramos del vano mandibular. Estuvo dado en primer lugar por el momento desde el voladizo $\mathrm{C}_{1}-\mathrm{A}$, que resulta de la siguiente ecuación. Donde $\mathrm{FC}_{1} \mathrm{y} \mathrm{FPm}_{4}$ son las fuerzas canina y carnasial unilaterales (i.e. consideradas en una de las ramas laterales de la mandíbula, siendo componentes parciales de FMC y FMM respectivamente), ubicadas en el plano antero posterior del cráneo de $P$. onca (sensu Del Moral et al. 2009):

$$
\begin{gathered}
\text { Mto. Máx. } \mathrm{A}_{\text {voladizo }}[\mathrm{Ncm}]=\left(-\mathrm{FC}_{1} \mathrm{~L}_{1}\right)+\left(-\mathrm{FPm}_{4} \mathrm{LPm}_{4}\right)+\left(-\mathrm{q}_{1} \mathrm{~L}_{1}^{2} / 6\right)+\left(-\mathrm{q}_{2} \mathrm{~L}_{1}^{2} / 8\right) \\
\mathrm{FC}_{1}=1 / 2 \mathrm{FMC} ; \mathrm{FPm}_{4}=1 / 2 \mathrm{FMM}
\end{gathered}
$$

El momento desde el apoyo móvil B al A, queda definido por:

$$
\text { Mto. Máx. B-A }[\mathrm{Ncm}]=\left(-\mathrm{q}_{3} \mathrm{~L}_{3} 2 / 8\right)
$$

En tanto para los pecaríes se calcularon las tensiones externas e internas de compresión y tracción (a las que se encuentran sometidas al aplicar el jaguar su $\mathrm{FC}_{1}$ ) en una sección de corte S-S del neurocráneo basal (Fig. 3b) mediante el "Método de los nudos de Cremona" (véase Badoux 1986, Callister 2000). El área de acción de $\mathrm{FC}_{1}$ se estandarizó en la región medial de los temporales en los pecaríes, en función del ángulo de apertura mandibular $\left(65^{\circ}\right)$ del jaguar cuando muerde inicialmente, y evidencia indirecta de campo sobre cráneos perforados y fracturados; durante la depredación hacia estas especies-presas.

Análisis de los datos. En el tratamiento de los datos se empleó el método de aproximaciones sucesivas de Cross (Cross 1949, Timoshenko 1983, Falter 1998, Eaton 2001, Del Moral et al. 2009). Se calcularon, graficaron y compararon las reacciones, momentos flexores y esfuerzos de corte en los dos tramos del vano mandibular de $P$. onca. A los efectos de optimizar este análisis se promediaron las medidas cráneo dentarias y los valores absolutos de FMC y FMM obtenidas en base a los especímenes de la muestra y las medidas craneométricas reportadas en la literatura consultada. En este punto, el cálculo y mapeo del estrés mandibular se efectúo a nivel de especie. Sin discriminar entre subespecies o fenotipos, como se realizara anteriormente con las fuerzas de mordedura, por considerarse carente de relevancia las diferencias de momentos flexores y esfuerzos de corte (debido a la similitud de forma y función del cráneo y la rama mandibular) entre los diferentes fenotipos de $P$. onca. 


\section{RESULTADOS}

Fuerzas de mordedura. Las formas de $P$. onca con mayores fuerzas caninas fueron en primer lugar $P$. o. palustris (presente desde Mato Grosso en Brasil hasta el Norte de la Argentina con $800 \mathrm{~N}$ ), seguido por P.o. centralis (que se distribuye desde el norte de Colombia hasta Costa Rica con 774,09 N) y P. o. onca (de la cuenca del río Amazonas y tributarios hasta Venezuela y Guyana con 723,86 N). Sin embargo en cuanto a la fuerza carnasial, no necesariamente se encontró la misma tendencia. $P$. $o$. palustris $(2360,96 \mathrm{~N})$ tuvo la mayor FMM seguido por el casi extinto $P$. o. veraecrucis (distribuido en el pasado desde el Este de México hasta el centro de Texas en Estados Unidos con $2320 \mathrm{~N})$ y $P$. o. onca $(2318,80 \mathrm{~N})$. En tanto los fenotipos de jaguar con menores fuerzas mandibulares, correspondieron a $P$. $o$. hernandesii (de la costa del Pacífico mexicano con 524,86 N) que tuvo la menor fuerza canina y P.o. centralis $(1638 \mathrm{~N})$ con la menor fuerza carnasial (Fig. 4, véase también Cuadro 3). En promedio se encontró que la fuerza carnasial fue 3 veces mayor, respecto a la fuerza canina en condiciones similares de mordedura. No obstante entre las diferentes subespecies

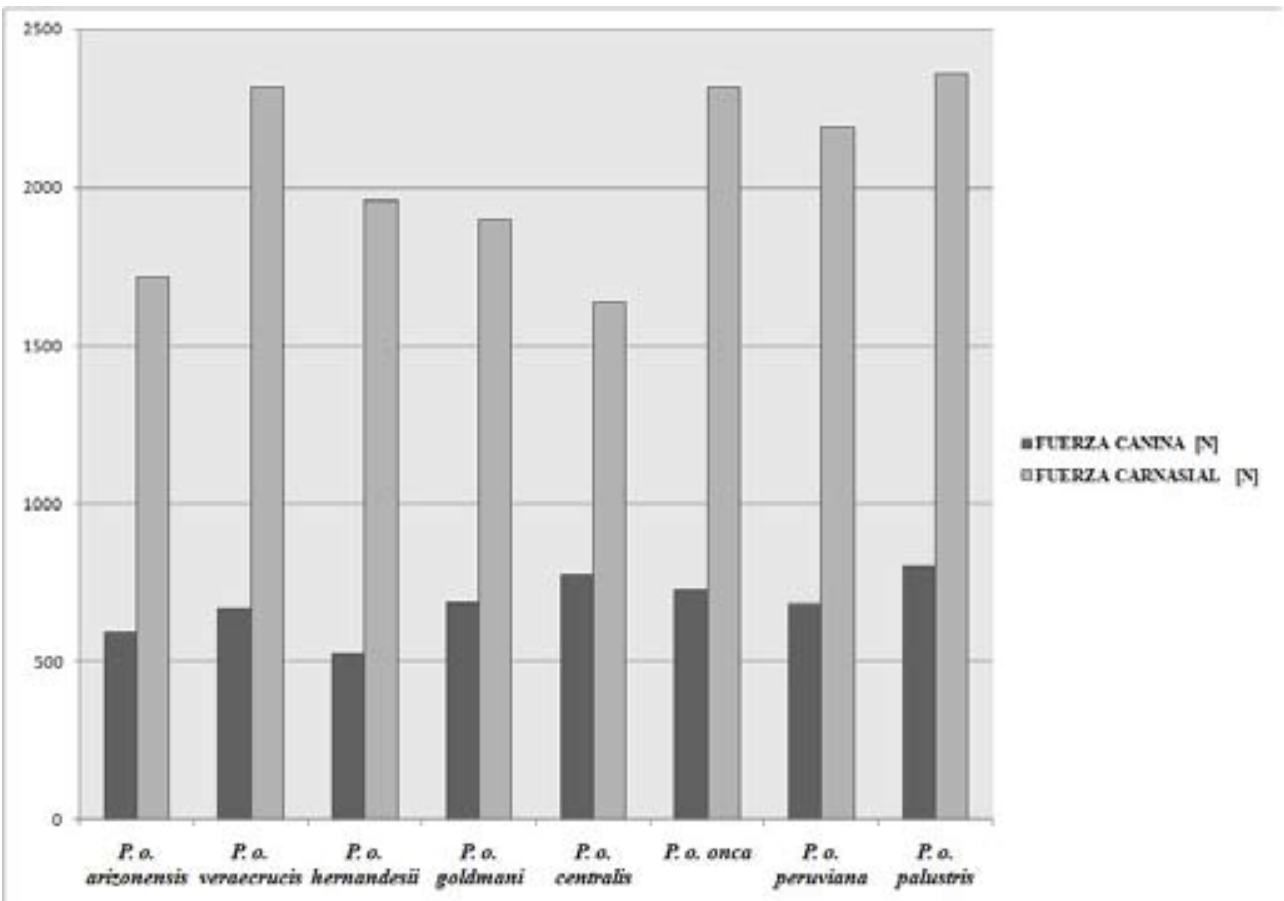

Figura 4. Comparación de fuerzas (en Newton) de mordedura canina (FMC) y fuerzas de mordedura carnasial (FMM), entre diferentes subespecies o fenotipos de Panthera onca (véase también el Cuadro 3). 
Del Moral Sachetti et al.: Mecánica de mordedura del jaguar durante la depredación de pecaríes

Cuadro 3. Comparación de valores absolutos (en Newtons) de la fuerza de mordedura derivada de los caninos (FMC) con la fuerza de mordedura derivada de los carnasiales (FMM) entre diferentes subespecies o fenotipos de Panthera onca.

\begin{tabular}{ccc}
\hline Subespecie & Fuerza Canina $[\mathrm{N}]$ & Fuerza Carnasial $[\mathrm{N}]$ \\
\hline P. o. arizonensis & 593,52 & 1715,90 \\
P. o. veraecrucis & 668,74 & 2320,00 \\
P. o. hernandesii & 524,86 & 1960,44 \\
P. o. goldmani & 686,64 & 1897,20 \\
P. o. centralis & 774,09 & 1638,00 \\
P. o. onca & 723,86 & 2318,80 \\
P. o. peruviana & 680,80 & 2192,32 \\
P. o. palustris & 800,00 & 2360,96 \\
\hline
\end{tabular}

esta relación fue disímil, teniendo una relación máxima de 3,74 en $P$. o. hernandesii y una relación mínima de 2,11 en P.o. centralis.

Estrés craneal en los pecaríes. La $\mathrm{FC}_{1}$ del jaguar aplicada en el hueso temporal (considerada en uno de los lados laterales del cráneo; Fig. 3b) de los pecaríes, produjo un esfuerzo compresivo que ascendió desde $36 \mathrm{~N}$ en $T$. pecari (Fig. $5 \mathrm{~b}$ ) a $42 \mathrm{~N}$ en $C$. wagneri (Fig. $5 \mathrm{c}$ ). Asimismo se encontró que las mayores tensiones, correspondieron a esfuerzos de tracción en el proceso yugular. En C. wagneri y T. pecari este esfuerzo ascendió a $164 \mathrm{~N}$ y en $P$. tajacu fue de $161 \mathrm{~N}$ (Fig. 5a). En consecuencia, el cóndilo occipital, el occipucio y los septos interalveolares del neurocráneo basal de los tayassuidos se encontraron sometidos a compresión (Cuadro 4). Asimismo, el hueso fresco tiene una resistencia a la compresión de $\sim 1054 \mathrm{~N} / \mathrm{cm}^{2}$ (Evans 1957). En un punto de contacto promedio de $0,89 \mathrm{~cm}^{2}$ del hueso con el canino durante la mordida máxima (la presión de la fuerza del jaguar en conjunto), producirá un esfuerzo de $\sim 681,56 \mathrm{~N}$ que provocaría en primera instancia la perforación del hueso plano en el pecarí. El perfil de las fuerzas de mordida en $P$. onca va incrementándose (en los premolares), a medida que el brazo de palanca aumenta y el ángulo de abertura mandibular disminuye (desde $65^{\circ}$ a $05^{\circ}$ ). En la serie carnasial, la tensión compresiva provocada por FMM ascendería a $\sim 5600 \mathrm{~N}$, mediante la cual el hueso llega a colapsar totalmente. Por último, el modelo básico del neurocráneo (en sección transversal con el refuerzo de los septos interalveolares) en los pecaríes, responde a un reticulado del tipo "súper abundante". Donde el número de barras concurrentes a los respectivos nudos ó vértices del reticulado, supera el necesario para mantener estricta rigidez. Así en las 3 especies de pecaríes, se cumple la relación: $n^{\circ}$ de barras $>2$ nudos -3 (véase Fig. 5).

Estrés mandibular en el jaguar. En $P$. onca se encontró mediante el método y 


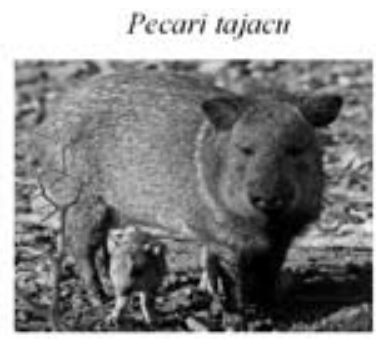

A

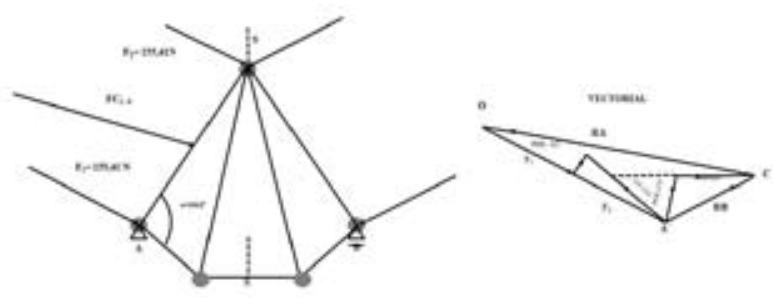

Tayassu pecari
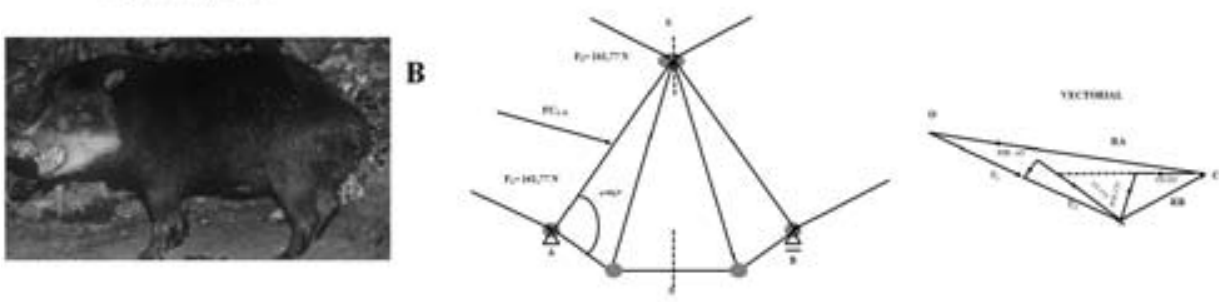

Catagonus wagneri
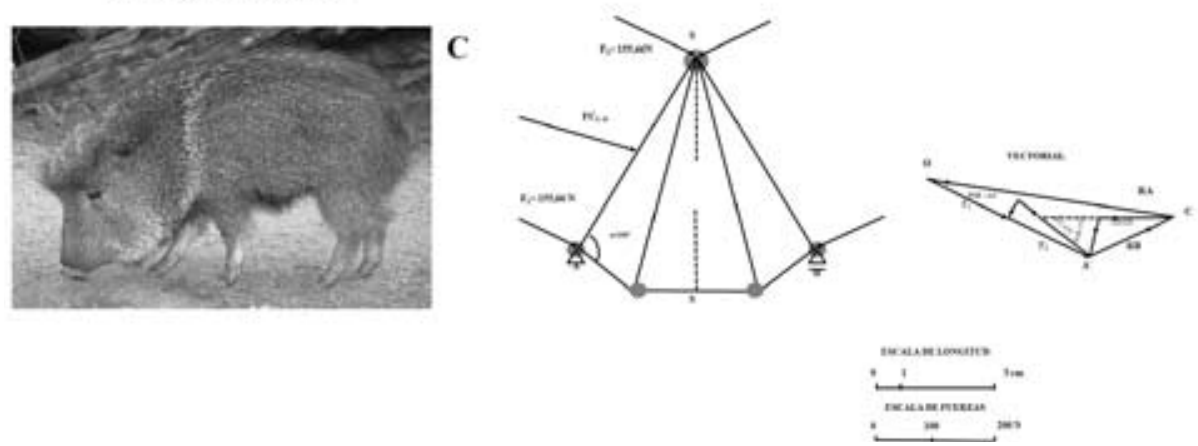

Figura 5. Diagrama de los nudos de Cremona sobre las tensiones externas e internas (en Newton), del neurocráneo de las 3 especies de pecaríes, durante la aplicación de la fuerza canina unilateral $\left(\mathrm{FC}_{1}\right)$ del jaguar. A) Pecari tajacu; B) Tayassu pecari; C) Catagonus wagneri (véase explicación en el texto y también el Cuadro 4).

diagrama de Cross, que hubo diferencias significativas de intensidades en reacciones a las cargas, momentos flexores y esfuerzos de corte que actúan sobre la línea normal (altura mandibular respectiva en los apoyos) del inicio de la fosa masetérica y la apófisis condilar (Fig. 6a). 
Cuadro 4. Valores máximos de las tensiones externas e internas (en Newtons) en el neurocráneo de Pecari tajacu, Tayassu pecari y Catagonus wagneri durante la aplicación de la fuerza canina unilateral

$\left(\mathrm{FC}_{1}\right)$ del jaguar. Por convención de signos se considera la compresión, negativa (-) y la tracción, positiva (+, véase explicación en el texto).

\begin{tabular}{cccc}
\hline Barra & P. tajacu & T.pecari & C. wagneri \\
\hline POE-AY & -40 & -36 & -42 \\
AY-CO & 161 & 164 & 164 \\
POE-CO & -81 & -84 & -77 \\
CO-CO & -116 & -109 & -132 \\
\hline
\end{tabular}

Los momentos máximos sobre la longitud del dentario fueron negativos y se encontraron en el tramo $\mathrm{C}_{1}$-A, a nivel de los carnasiales con $1375 \mathrm{Ncm}$. Alcanzando el máximo en el inicio de la fosa masetérica con $5466,11 \mathrm{Ncm}$. Los momentos mínimos fueron positivos y se localizaron en el tramo A-B, con 1,71 $\mathrm{Ncm}$ en A. El diagrama de momentos (Fig. 6b) muestra que la mandíbula se encuentra flexionada describiendo un decremento lineal (de ligera tendencia parabólica influenciado por el peso propio de la cabeza) en el $46 \%$ de la longitud total del dentario. En el $15,11 \%$ de la rama mandibular, la flexión se acentúa marcadamente producto de la inserción y acción normal del músculo masetérico. En todo el tramo $\mathrm{C}_{1}$-A, la mandíbula se encuentra sometida por intensos esfuerzos de tracción. Seguidamente en el tramo A-B, parcialmente las crestas del coronoides $(14,71 \%)$ y las crestas del proceso angular $(24,18 \%)$ tienen tensiones despreciables de tracción; generadas por el momento flexor produci-

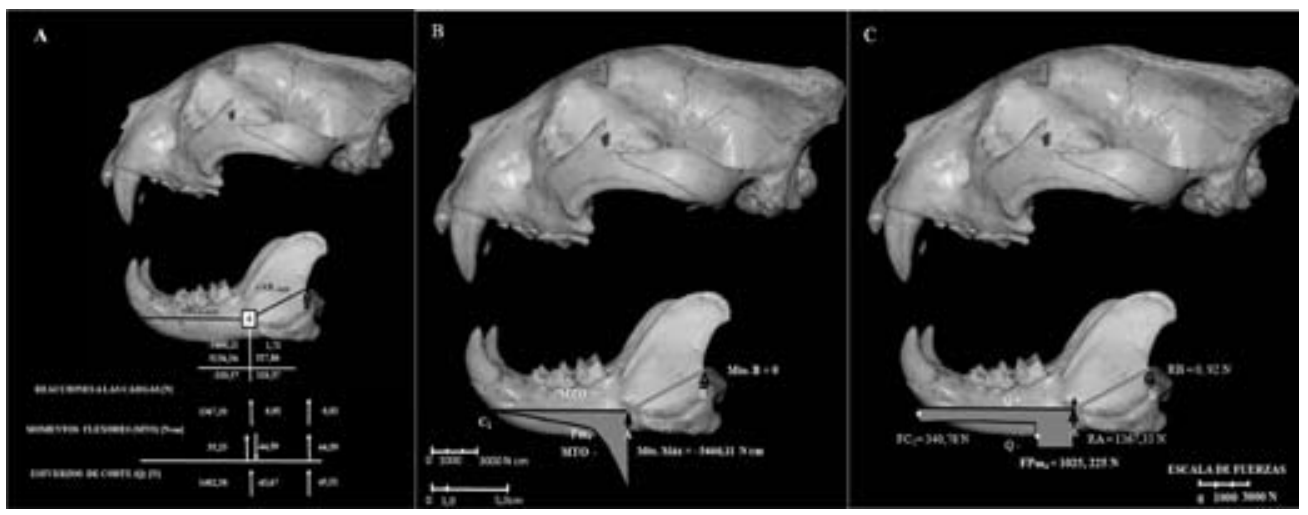

Figura 6. Diagrama de aproximaciones sucesivas de Cross, momentos flexores y esfuerzos de corte sobre el dentario de Panthera onca. A) Obtención de las reacciones a las cargas, momentos flexores y esfuerzos de corte en el inicio de la fosa masetérica (A). B) y C) Diagramas de momentos flexores y esfuerzos de corte (véase explicación en el texto). 
do por las componentes normales y de retracción del complejo masetérico-temporal, durante la mordedura máxima (véase Fig. 6b).

En tanto, las mayores fuerzas cortantes fueron las producidas por $\mathrm{FC}_{1}(340,78 \mathrm{~N})$ en el canino y $\mathrm{FPm}_{4}(1025,225 \mathrm{~N})$ en el carnasial. La reacción vertical en el masetérico (apoyo $\mathrm{A} ; 1367,33 \mathrm{~N}$ ) que resiste el corte de $\mathrm{FC}_{1}, \mathrm{FPm}_{4} \mathrm{y}$ el del peso propio de la cabeza; fue $\sim 1486,23$ veces mayor respecto a la apófisis condilar (apoyo B; 0,92 N). Donde los esfuerzos de corte son positivos y despreciables sobre la rama ascendente de la fosa masetérica (Fig. 6c).

\section{DISCUSIÓN}

El jaguar depreda sobre presas que tienen una menor o igual masa corporal, hasta presas que inclusive pueden llegar a quintuplicarlo en tamaño (e.g. ganado doméstico y caimanes; véase Leyhausen 1979, Schaller \& Crawshaw 1980, Rabinowitz 1986, Crawshaw \& Quigley 2002, Da Silveira et al. 2010). Los mamíferos-presa de $P$. onca presentan frecuentemente una mordida en la base del cráneo detrás de las orejas o en la nuca (con rotura del neurocráneo), fracturas de las vértebras cervicales e infrecuentemente las presas más grandes son asfixiadas mediante una mordedura en la tráquea (obs. pers.). Los resultados obtenidos en este trabajo, sugieren que las diversas subespecies o fenotipos reconocidos de jaguar, tienen fuerzas de mordida canina y carnasial altamente compresivas para provocar la perforación y fractura del neurocráneo en los pecaríes (una de las presas principales de $P$. onca) durante la depredación y remoción ósea, para acceder al contenido graso intra-óseo de la cabeza. La intensidad de la mordedura posibilita además, la destrucción y el aprovechamiento de diversos tejidos duros en diferentes presas; como el cráneo de carpinchos (Hydrochaeris hydrochaeris) y de cérvidos, caparazones de tortugas, integumentos de armadillos y caimanes, entre otros (Schaller \& Vasconcelos 1978, Emmons 1989, Sunquist \& Sunquist 2002, Meachen-Samuels \& Van Valkenburgh 2009). Como otros félidos, $P$. onca tiende a no consumir huesos.

La fractura de huesos requiere de la transferencia de estrés altamente compresivo, en el punto de contacto de los dientes y el hueso durante la mordedura. En los pantherines en general y el jaguar en particular, el gran desarrollo cigomático-mandibular (en relación a su masa corporal) posibilita el incremento de la fuerza canina por unidad de superficie, en el punto de contacto con el hueso. Los caninos robustos de $P$. onca con una cúspide más roma (comparados con caninos de félidos de tallas menores) producen una transferencia distribuida de la fuerza canina, lo que reduce su intensidad. En contraposición los gatos de tallas menores, al tener menor punto de contacto entre diente y hueso, la fuerza canina por unidad de área aumenta. Esto posibilita un óptimo esfuerzo de corte para penetrar piel y huesos, con menor requerimiento de fuerza cigomático-mandibular (Frazzetta 1988, Freeman \& Lemen 2007, MeachenSamuels \& Van Valkenburgh 2009). También en el jaguar, el esfuerzo compresivo 
en sus caninos (producto de la musculatura aductora), causa en estos dientes flexión axial o pandeo; que precede a la fractura por compresión simple. Nosotros hemos estimado conservadoramente la fuerza canina, en función de la resistencia teórica de los caninos a pandeo (véase Fuerza Canina en Fig. 4 y Cuadro 3). Esto implica que $P$. onca puede inclusive aplicar mayor fuerza durante la mordedura, pero esto produciría la fractura del diente. Una estrategia evolutiva que ha reducido la probabilidad de fractura de los caninos en carnívoros, es la menor relación existente entre la longitud del canino respecto a su diámetro (Rensberger 1999). Cuando la longitud $\left(\mathrm{C}_{1} \mathrm{H}\right)$ supera a su ancho mínimo $\left(\mathrm{C}_{1} \mathrm{ML}\right)$ en una relación $\mathrm{C}_{1} \mathrm{H}>30 \mathrm{C}_{1} \mathrm{ML}$ la deformación por pandeo resulta muy evidente. En $P$. onca la relación encontrada en general es $\sim 2$ (véase dimensiones de caninos en Cuadro 1) esto denota la rigidez de los caninos y su resistencia a una carga y flexión axial de $\sim 340 \mathrm{~N}$. En tanto, los premolares de forma piramidal con amplia base y bajo perfil, son resistentes 3 veces más a la compresión simple respecto a los caninos. En este punto se observa una correlación, con la perforación/remoción de huesos del neurocráneo en los pecaríes. Así, se ha encontrado que el neurocráneo de los tayassuidos responde a un modelo reticulado triangular de extrema rigidez, cuya atrición requiere gran presión del canino o los carnasiales en el hueso. Aunque las estimaciones de los esfuerzos internos del neurocráneo en los pecaríes, implica según premisas estáticas, que el reticulado se encuentra en equilibrio; se puede considerar entonces que estas tensiones son válidas, solo segundos previos a la perforación o rotura. Pues finalmente el sistema colapsa, mediante la destrucción sustancial del cráneo y nasales (como se ha observado en carcasas consumidas de pecaríes encontradas en campo, Del Moral obs. pers.).

En individuos de $P$. onca de vida libre, algunas patologías periodontales (e.g. gingivitis, cálculo dental, retracción gingival, bolsa periodontal) podrían condicionar las fuerzas de mordedura y la resistencia a pandeo o compresión simple de caninos y carnasiales. Mediante la prevalencia de la dolencia dentaria y la consiguiente destrucción de los tejidos de soporte del diente. Asimismo, la mala oclusión puede generar cargas con diversas excentricidades durante la mordida y la masticación ortal, que pueden inducir un mayor riesgo de fractura de los dientes (obs. pers.). Restringiendo la eficiencia de la mordedura de $P$. onca para la cacería de sus presas naturales, provocando estos factores entre otros, la aparición del conflicto entre el jaguar y pobladores humanos debido a la depredación de ganado doméstico (presas potenciales que se presentan más vulnerables a la depredación con respecto a sus presas naturales) en mosaicos de áreas boscosas y pecuarias (véase Jackson \& Nowell 1996). Sin embargo, aparentemente la prevalencia natural de dolencias periodontales y mala oclusión es hasta 3 veces menor en individuos de vida libre, respecto a ejemplares de cautiverio (Rossi Junior et al. 2003, 2007). En cambio en este sentido, se ha documentado que individuos confirmados como predadores de ganado y posteriormente cazados por ganaderos, tenían heridas cráneo dentales antiguas originadas mediante disparos 
oportunistas de armas de fuego (Rabinowitz 1986, Hoogesteijn \& Mondolfi 1992, Jackson \& Nowell 1996, Leite et al. 2002).

Por último, en el dentario del jaguar se ha observado una correlación positiva entre el locus de momentos flexores y esfuerzos de corte máximos, y el aumento respectivo de la sección transversal de la mandíbula (entre $\mathrm{Pm}_{4}-\mathrm{M}_{1}$ y $\mathrm{Pm}_{3}-\mathrm{Pm}_{4}$, véase Fig. 6 b y $6 \mathrm{c}$ ). Este aumento de sección como el acortamiento de la rama mandibular, le confieren al dentario mayor resistencia mecánica a la flexión y corte durante la mordedura. Asimismo, el mayor rango de apertura mandibular denota una relación recíproca, con la funcionalidad del brazo de palanca y fuerzas de mordedura. A medida que disminuyen los ángulos de apertura mandibular al morder o masticar, el brazo de palanca del dentario aumenta con el consiguiente incremento de las fuerzas. Estas variables encontradas, coinciden con lo reportado por otros autores (véase Thomason 1991, Wroe et al. 2005, Christiansen 2007, 2008, Mc Henry et al.2007, MeachenSamuels \& Van Valkenburgh 2009), sobre la mecánica de mordedura en grandes félidos y cánidos; considerándose caracteres diagnósticos importantes de especialistas en grandes presas (Salles 1992, Christiansen 2007, 2008, Meachen-Samuels \& Van Valkenburgh 2009). Particularmente en los pantherines, estas ventajas mecánicas aparentemente, han influenciado la evolución del cráneo en el taxón. Produciendo mayor amplitud de los arcos cigomáticos (que félidos de tallas menores), la elevación y el alargamiento de la porción caudal del cráneo. Permitiendo como causa última, el aumento de la musculatura aductora (Gittleman 1986, Christiansen 2008, MeachenSamuels \& Van Valkenburgh 2009).

AGRADECIMIENTOS. Al personal, y directivos de la Estación Biológica de Fauna Silvestre Dr. Martín Vucetich (Jujuy, Argentina), Colección Boliviana de Fauna (La Paz, Bolivia), Museo de Historia Natural Noel Kempff Mercado (Santa Cruz de la Sierra, Bolivia), Zoológico Miguel Álvarez del Toro (Chiapas, México) y el Museo de Historia Natural de la Universidad Nacional Mayor de San Marcos (Lima, Perú) por su hospitalidad; y permitirnos acceder a los ejemplares del estudio. Se agradece a Belén Etchegaray, por su colaboración con las fotografías de jaguar que ilustran el presente. Sin dejar de mencionar a Marcelo Stucchi (Perú), quien facilitó, dimensiones de un especímen de este país. También se reconoce de Argentina: Arturo A. Canedi y Jorge Moreno. En especial por su apoyo a Roberto F. Del Moral, Angela F. Sachetti y particularmente Alcira M. Contreras. Finalmente se dedica el presente estudio al Dr. Arturo A. Canedi (Jujuy, Argentina). Dos revisores anónimos hicieron comentarios que permitieron mejorar el artículo.

\section{LITERATURA CITADA}

Aparicio, P.M. \& C. López Plana. 2011. Atlas de anatomía de especies silvestres de la Amazonia peruana. Universitat Autònoma de Barcelona. http://atlasanatomiaamazonia.uab.cat/ (visitado: 0103-2010).

Aranda, M. 1992. El jaguar (Panthera onca) en la Reserva Calakmul, México: morfometría, hábitos alimentarios y densidad de población, pp. 235-274. In: R. Clemente (Eds.). Felinos de Venezuela: biología, ecología y conservación. Fudeci, Valencia. 
Aranda, M. 1994. Importancia de los pecaríes (Tayassu spp.) en la alimentación del jaguar (Panthera onca). Acta Zoológica Mexicana (n.s.), 62:11-22.

Badoux, D. M. 1986. Biostática y biodinámica, pp.47-49. In: Sisson, S. \& R. Getty (Eds.). Anatomía de los animales domésticos ( $4^{a}$ Edición). Salvat, Barcelona.

Binder, W. J. \& B. Van Valkenburgh. 2000. Development of bite strength and feeding behavior in juvenile spotted hyena (Crocuta crocuta). Journal of Zoology (London), 252: 273-283.

Brock, S. E. 1963. The jaguar (Panthera onca). Journal of the British Guiana Museum and Zoo, 37: 46-48.

Callister, W. D. 2000. Ciencia e ingeniería de los materiales. Reverté, Barcelona.

Carrillo, E. 2000. Ecology and conservation of white-lipped peccaries and jaguars in Corcovado National Park, Costa Rica. Tesis PhD, University of Massachusetts, USA.

Cavalcanti, S. 2008. Predator-prey relationships and spatial ecology of jaguars in the Southern Pantanal, Brazil: implications for conservation and management. Tesis $\mathrm{PhD}$, Utah State University, Logan, Utah.

Chinchilla, F. 1994. La dieta del jaguar (Panthera onca), el puma (Felis concolor) y el manigordo (Felis pardalis) (Carnivora: Felidae) y dos métodos de evaluación de su abundancia relativa en el Parque Nacional Corcovado, Costa Rica. Tesis de Maestría, Universidad Nacional de Heredia, Costa Rica.

Christiansen, P. 2007. Canine morphology in the larger Felidae: implications for feeding ecology. Biological Journal of the Linnean Society, 91: 573-592.

Christiansen, P. 2008. Evolution of skull and mandible shape in cats (Carnivora: Felidae). PLoS ONE, 3: e2807.

Christiansen, P. \& S. Wroe. 2007. Bite forces and evolutionary adaptations to feeding ecology in carnivores. Ecology, 88: 347-358.

Crawshaw, P. G. \& H. B. Quigley. 2002. Hábitos alimentarios del jaguar y el puma en el Pantanal, Brasil, con implicaciones para su manejo y conservación, pp.223-235. In: A. B. Taber, C.B. Chetkiewicz, R. Medellin, A. Rabinowitz \& K.H. Redford (Eds.). El jaguar en el nuevo milenio. Fondo de Cultura Económica, Universidad Nacional Autónoma de México \& Wildlife Conservation Society, Distrito Federal, México.

Cross, H. 1949. Analysis of continuos frames by distributing fixed-ed moments, pp.1-12. In: L. B. Grinter (Eds.). Numerical Methods of Analysis in Engineering, Successive corrections. McMillan Company, New York.

Da Silveira, R., E. E. Ramalho, J. B. Thorbjarnarson \& W. E. Magnusson. 2010. Depredation by jaguars on caimans and importance of reptiles in the diet of jaguar. Journal of Herpetology, 44: 418-424.

Dechow, P. C. \& D. S. Carlson. 1983. A method of bite force measurement in Primates. Journal of Biomechanics, 16: 797-802.

Del Moral S., J.F., R. Zenteno C. \& F. I. Lameda C. 2009. Análisis biostático de las tensiones actuantes en el dentario del oso andino (Tremarctos ornatus) durante la mordedura. Acta Zoológica Mexicana (n.s.), 25: 551-567.

Eaton, L. K. 2001. Hardy Cross and the moment distribution method. Nexus Network Journal, vol. 3, $\mathrm{N}^{\mathrm{o}}$ 2. http://www.nexusjournal.com/Eaton.html

Emmons, L. H. 1987. Comparative feeding ecology of felids in a Neotropical forest. Behavioral Ecology and Sociobiology, 20: 271-283.

Emmons, L. H. 1989. Jaguar predation on chelonians. Journal of Herpetology, 23:311-314.

Emmons, L. H. 1992. Tamaño corporal y estrategias de alimentación, pp.246. In: J. Seidensticker \& S. Lumpkin (Eds.). Felinos. Encuentro Editorial S. A., Barcelona. 
Evans, F. G. 1957. Stress and strain in bones: their relation to fractures and osteogenesis. Charles C. Thomas, Springfield.

Ewer, R. F. 1973. The carnivores. Cornell University Press, New York.

Falter, H. 1998. The influence of mathematics on the development of structural form, pp. 51-64. In: Kim Williams (Eds.). Nexus II: Architecture and Mathematics. Edizioni dell'Erba Fucecchio, Florence.

Frazzeta, T. H. 1988. The mechanics of cutting and the form of shark teeth (Chondrichthyes, Elasmobranchii). Zoomorphology, 108: 93-107.

Freeman, P. W. \& C. A. Lemen. 2007. The trade-off between tooth strength and tooth penetration: predicting optimal shape of canine teeth. Journal of Zoology (London), 273: 273-280.

González-Maya, J. F. 2007. Densidad, uso de hábitat y presas del jaguar (Panthera onca) y el conflicto con humanos en la región de Talamanca, Costa Rica. Tesis de Maestría, Centro Agronómico Tropical de Investigación y Enseñanza, Turrialba, Costa Rica.

Gittleman, J. L. 1986. Carnivore brain size, behavioral ecology and phylogeny. Journal of Mammalogy, 67: 23-36.

Grubb, P. 2005. Family Tayassuidae, pp. 643-644. In: Wilson, D.E. \& D.M. Reeder (Eds.). Mammals Species of the World. John Hopkins University Press, Baltimore.

Grubb, P. \& C.P. Groves. 1993. The Neotropical tayassuids Tayassu and Catagonus: Taxonomy and descriptions, pp. 5-7. In: Oliver, W. L. R. (Eds.). Pigs, Peccaries and Hippos. Status Survey and Conservation Action Plan. IUCN, Gland.

Hoogesteijn R. \& E. Mondolfi. 1992. El jaguar, tigre americano. Armitano Editores C.A., Caracas.

Hoogesteijn R. \& E. Mondolfi. 1996. Body mass and skull measurements in four jaguar populations and observations on their prey base. Bulletin Florida Museum Natural History, 39: 195-219.

Hoogesteijn R. \& A. Hoogesteijn. 2008. Conflicts between cattle ranching and large predators in Venezuela: could use of water buffalo facilitate felid conservation? Oryx, 42: 132-138.

Jackson, P. \& K. Nowell. 1996. Problems and possible solutions in management of felid predators. Journal of Wildlife Research, 1: 304-314.

Kiltie, R. A. 1982. Bite force as a basis for niche differentiation between rain forest peccaries (Tayassu tajacu and T. pecari). Biotropica, 14: 188-195.

Leite, M. R., R. L. Boulhosa, F. Galvão \& L. Cullen. 2002. Conservación del jaguar en las áreas protegidas del Bosque Atlántico de la costa de Brasil, pp.25-42. In: A. B. Taber, C. B. Chetkiewicz, R. Medellin, A. Rabinowitz \& K. H. Redford (Eds.). El jaguar en el nuevo milenio. Fondo de Cultura Económica, Universidad Nacional Autónoma de México \& Wildlife Conservation Society, Distrito Federal, México.

Leyhausen, P. 1979. Cat behavior: the predatory and social behavior of domestic and wild cats. Garland STMP Press, New York.

McHenry, C. R., S. Wroe, P. D. Clausen, K. Moreno \& E. Cunningham. 2007. Super modeled saber cat, predatory behavior in Smilodon fatalis revealed by high-resolution 3D computer simulation. PNAS, 104: 16010-16015.

McMahon, T. A. 1975. Allometry and biomechanics: limb bones in adult ungulates. American Naturalist, 109.

Meachen-Samuels, J. \& B. Van Valkenburgh. 2009. Craniodental indicators of prey size preference in the Felidae. Biological Journal of the Linnean Society, 96: 784-799.

Nomina Anatomica Veterinaria. 2005. International Committee on Veterinary Gross Anatomical Nomenclature \& General Assembly of the Veterinary Anatomist (Eds.). Editorial Committee, Hannover, Columbia.

Pocock, R. I. 1939. The races of jaguar (Panthera onca). Novitates Zoologicae, 41: 406-422. 
Rabinowitz, A. R. 1986. Jaguar predation on domestic livestock in Belize. Wildlife Society Bulletin, 14: 170-174.

Rabinowitz, A. R. \& B.G. Nottingham. 1986. Ecology and behavior of the jaguar (Panthera onca) in Belize, Central America. Journal of Zoology (London), 210: 149-159.

Rensberger, J. M. 1999. Enamel microstructural specialization in the canine of the spotted hyena, Crocuta crocuta. Scanning Microscopy International, 13: 343-361.

Rossi Junior, J. L., M. A. Gioso, J. C. Ramos da Silva \& M. F. Vianna Marvulo. 2003. Prevalência de maloclusão em Panthera onca e Puma concolor mantidas em cativeiro no Estado de São Paulo. Brazilian Journal of Veterinary Research and Animal Science, 40: 155-160.

Rossi Junior, J. L., M. A. Gioso \& L. M. Domingues-Falqueiro. 2007. Estudo comparativo sobre prevalênciade doença periodontal em Panthera onca mantida em cativeiro e em indivíduos de natureza. Pesquisa Veterinária Brasileira, 27: 209-214.

Salom-Pérez, R., E. Carrillo, J. C. Sáenz \& J. M. Mora. 2007. Critical condition of the jaguar Panthera onca population in Corcovado National Park, Costa Rica. Oryx, 41: 51-56.

Salles, L.O. 1992. Felid phylogenetics: Extant taxa and skull morphology (Felidae, Aeluroidea). American Museum Novitates, 3047: 1-67.

Schaller, G. B. 1983. Mammals and their biomass on a Brazilian ranch. Arquivos de Zoologia, 31: 136.

Schaller, G. B. \& P.G. Crawshaw. 1980. Movement patterns of jaguar. Biotropica, 12: 161-168.

Schaller, G. B. \& J.M.C. Vasconcelos. 1978. Jaguar predation on capybara. Zeitschrift Säugetierk, 43: 296-301.

Seymour, K. L. 1989. Panthera onca. Mammalian Species, 340: 1-9.

Sunquist, M. \& F. Sunquist. 2002. Wildcats of the world. University of Chicago Press, Chicago.

Therrien, F. 2005. Mandibular force profiles of extant Carnivorans and implications for the feeding behaviours of extinct predators. Journal of Zoology (London), 267: 249-270.

Thomason, J. J. 1991. Cranial strength in relation to estimated biting forces in some mammals. Canadian Journal of Zoology, 69: 2326-2333.

Timoshenko, S. 1983. History of the strength of materials, with a brief account of the history of theory of elasticity and theory of structures. Mc Graw-Hill, New York.

Wetzel, R. M. 1977. The Chacoan peccary, Catagonus wagneri (Rusconi). Bulletin of Carnegie Museum of Natural History, 3: 1-36.

Wroe, S., C. McHenry \& J. J. Thomason. 2005. Bite club: Comparative bite force in big biting mammals and the prediction of predatory behavior in fossil taxa. Proceedings of the Royal Society (London), 272: 619-625. 